\title{
Psycho-behavioral factors associated with neurocognitive performance among people living with HIV on antiretroviral therapy in Accra, Ghana
}

\author{
Nana Asiedu ${ }^{1}$, Irene Kretchy ${ }^{2}$ Emmanuel Asampong ${ }^{1}$
}

1. University of Ghana College of Health Sciences, Social and Behavioural Sciences, School of Public Health

2. University of Ghana College of Health Sciences, Pharmacy Practice and Clinical Pharmacy

\begin{abstract}
Background: It is estimated that almost half of all people living with HIV have some form of neurocognitive impairment, but few studies have looked at the risk of neurocognitive impairment and its associated factors in Ghana, due in part to limited resources for such testing.

Objective: To examine neurocognitive performance in a group of Ghanaians living with HIV and possible factors that contribute to their performance.

Methods: One hundred and four patients were assessed using a selection of brief non-invasive neuropsychological assessments as well as the International HIV Dementia Scale. Psycho-behavioural factors (alcohol use, depression, and medication adherence) as well as demographic characteristics and functional daily activities were assessed to determine their association with neurocognitive performance, using linear regression and receiver operating characteristic analyses.

Results: About 48\% of the participants met the criteria for risk of neurocognitive impairment. Age, education, and symptoms of depression were found to be significantly associated with the risk of impairment.

Conclusion: Some people living with HIV showed risk of neurocognitive impairment, which was significantly associated with education, age and depressive symptoms. It is therefore important to consider routine neurocognitive screening in HIV management to recognize any risks for early interventions.

Keywords: ART adherence; depression; neurologic disease.

DOI: https://doi.org/10.4314/ahs.v20i2.6

Cite as: Asiedu N, Kretchy I, Asampong E. Psycho-behavioral factors associated with neurocognitive performance among people living with HIV on antiretroviral therapy in Accra, Ghana. Afri Health Sci. 2020; 20(2): 587-596. https://doi.org/10.4314/ahs.v20i2.6
\end{abstract}

\section{Introduction}

The Human Immunodeficiency Virus (HIV) compromises the immune system to cause Acquired Immune Deficiency Syndrome (AIDS) and has the capability to infect the central nervous system $(\mathrm{CNS})^{1}$. A major consequence of HIV infection in the brain, especially for patients without medication, is neurocognitive impairment $(\mathrm{NCI})$ or what are sometimes categorized as HIV Associated Neurocognitive Disorders (HAND) ${ }^{2,3}$. As viral load increases, NCI becomes more severe, with AIDS presenting the worst prognosis for cognition $^{4}$. The cognitive deficits can be global, but specific areas affected include psychomotor skills, speed of
Corresponding author:
Irene Kretchy,
University of Ghana
College of Health Sciences,
Pharmacy Practice and Clinical Pharmacy
Email: ikretchy@ug.edu.gh

information processing, memory, attention, language, and perception ${ }^{5}$. With the availability and access to antiretroviral therapy, progression of HIV can be curbed, corresponding with a reduction in neurocognitive impairment ${ }^{6}$. However, milder cases of HAND, such as asymptomatic neurocognitive impairment and mild neurocognitive disorder are reported to be prevalent in cases of undetectable viral $\operatorname{load}^{7}$. Almost half of all people living with HIV are estimated to have some form of impairment ${ }^{2}$.

Although sub-Saharan African countries account for more than $66 \%$ of the world's HIV population ${ }^{8}$, the implication of cognitive deficits due to HIV has not been extensively explored. In low and middle income countries, the prevalence of HAND ranges from $6 \%$ to $64 \%$ in adults on ART medication ${ }^{9}$. It is also important to establish that most HAND studies were conducted in the developed world, in which most patients have the HIV-1 sub-type B; HIV cases in Africa have the non-B HIV subtypes, and how this may affect the de- 
velopment of NCI is unclear ${ }^{8}$. In Ghana, there is an initiative to have $85 \%$ of eligible patients on ART to limit the number of AIDS-specific fatalities ${ }^{10}$. Where ART is limited, or where there is poor adherence to the medications, it is important to consider the possibility of severe cases of NCI. With an estimated 310, 000 Ghanaians living with HIV and about $42 \%$ of persons 15 years or older receiving and adhering to ART, milder forms of HAND may persist and cognitive impairment has the potential to adversely affect daily life ${ }^{11,12}$. To this end, this study sought to examine the pattern of neurocognitive performance in a sample of people living with HIV in Ghana, and to identify the factors that may contribute to the performance.

\section{Methods}

\section{Study design and site}

This was a cross-sectional, exploratory study, using questionnaires and neurocognitive tests to collect quantitative data from people living with HIV recruited from the HIV clinic at the Ridge Hospital in Accra, Ghana, during the month of June 2017. The HIV clinic at Ridge Hospital, situated in Accra, which is the capital and largest city of Ghana, regularly provides services such as HIV and STI testing and provides ART medications through the adjacent pharmacy. The clinic runs during the weekday, serving approximately 30 clients a week. The actual number of clients may vary depending on availability of medications. The client pool is mostly female, particularly those who come in-person to receive their medications. Ridge Hospital appeared to be an ideal place to recruit participants for an investigation, with the advantage that participants could be asked about their adherence to medications.

\section{Participants}

The study participants included adults (18 years and older) who had received a diagnosis of HIV and were on ART for about six months or more to be able to assess the level of adherence to the medications ${ }^{12}$. Based on the information in the health records, participants possible susceptibility to NCI such as having a prior diagnosis of a major psychiatric disorder like schizophrenia or major depressive disorder, or having a record of severe head injury, were excluded from the study. This was to ensure that no participant had any predisposition to NCI and to limit factors that could contribute to neurocognitive performance, independent of HIV.

Due to sampling challenges associated with hard-toreach study populations like persons with HIV and AIDS, all clients who attended the ART clinic during the study period were invited to participate in the study ${ }^{13}$. Persons who met the inclusion criteria and consented to be part of the study were recruited. In total 104 participants took part in the study.

\section{Measures}

All the materials were in the form of paper-based questionnaires and performance assessments. The questionnaire obtained the following characteristics from the participants: age, sex, current employment status, education level, and daily functional activities. In addition, the years the participant had been living with HIV, other conditions and current ART regimen were verified from health records.

The Psycho-behavioural profile for the participants were obtained using the Center for Epidemiologic Studies Short Scale Depression Scale (CES-D), Alcohol Use Disorders Identification Test (AUDIT), and the Questionnaire on Taking Anti-retroviral Medication (QTAM). The neurocognitive profile was obtained with the following assessments: Trail Making Test Part A (TMT A), Stroop Colour-Word (SCW), and the International HIV Dementia Scale (IHDS).

The CES-D is a 10-item depression scale, with points ranging from 0-3 assigned to each response. A participant obtaining a score of 10 or greater is considered to exhibit depressive symptoms. This test by itself does not constitute a clinical diagnosis for depression ${ }^{14}$.

The AUDIT is a 10-item screening tool developed by the World Health Organization to assess alcohol consumption, drinking behaviours, and alcohol-related problems and has been validated across gender, ethnic and racial groups. This study adopted the clinician-administered version with questions on the frequency of drinking behaviours. The scores for each participant was derived by adding the 10 items to give a total score ranging from $0-40$. Participants who had a score of 0 on the AUDIT abstained from alcohol use, those with a score of $1-7$ were non-hazardous drinkers and a score of 8 or more indicative of harmful alcohol use $\mathrm{e}^{15}$. The QTAM is a brief 5-item self-reported scale which asks participants in non-accusatory language about any tendencies to forget or opt not to take their medications. The scale included items that ask participants about the medications they take (if participants are unsure of names, medical records were used to confirm), and specific instances in which medications were not taken. Patients were considered non-adherent based on the items on the scale, if they reported not taking more than $5 \%$ of their pills on the preceding day, the penultimate day, the preceding seven days or during the past 30 days $^{16}$. 
The TMT is a timed neuropsychological test in which participants use a pen to connect circled numbers on paper. In Part "A," the participant is to draw lines to connect circled numbers in a numerical sequence (i.e., 1-2-3, etc.) as rapidly as possible until reaching the last number " 25 ". They are first given a sample to practice before proceeding to the actual test, in which their time to completion is measured. This test reflects a wide variety of cognitive processes in executive functioning: attention, visual search and scanning, sequencing and psychomotor speed. The TMT A has an inter-rater reliability coefficient of $0.99^{17}$.

The SCW test for this study focused on the ColourWord task, in which the individual is shown the names of colours printed in conflicting ink (e.g., the word "blue" in red ink) and is asked to name the colour of the ink rather than the word as quickly as possible. The Colour-Word task measures both mental flexibility and ability to inhibit a dominant response, because of the interference of the word is printed in a conflicting colour $^{18}$. The test-retest reliability coefficient for Colour Word is reported as $0.671^{19}$.

The IHDS is an internationally used tool for screening and identifying patients at risk of developing HAND in persons with HIV. The tool asks participants to perform memory and motor tasks and should take no longer than 10 minutes to complete. The tool starts with memory registration, in which four English words are read out loud to the patient and he/she is told to memorize them for later. The test then has three subsets: motor speed (patient taps first two fingers of non-dominant hand as quickly as possible), psychomotor speed (patient performs a specific sequence of hand movements with non-dominant hand as quickly as possible), and then memory recall (patient is asked to recall the four words that they were told during the memory registration). Each of the three subtests have a score range of 0 to 4 , creating a total range score of 0 to 12 . The IHDS uses a cut-off point of 11 to flag for dementia risk, but does not have specific metrics for milder cases of NCI which can be seen in HAND ${ }^{20}$.

The Katz Instrumental Activities of Daily Living questionnaire was used to confirm that the participants were not so disabled to require assistance in daily functioning. The index ranks adequacy of performance in the six functions: bathing, dressing, toileting, transferring, continence, and feeding. Clients were scored yes or no for independence in each of the six functions. A score of 6 indicates full function, 5 or 4 indicates moderate impairment, and 2 or less being severe functional impairment ${ }^{21}$.

\section{Ethical considerations}

Ethical approval was sought from the Ethical Review Committee of the Ghana Health Service (GHS-ERC: 48/12/16), the National AIDS Control Programme (NACP), as well as permission from the director of Ridge Hospital prior to the commencement of the study. Participants were told about the study and assured of confidentiality. Additionally, they were told that they could withdraw from the study at any time and doing so would not affect any of the services they received from the facility. Those who agreed to be part of the study were required to sign an informed consent form. Knowing that assessments may indicate (but not diagnose) dementia, the investigators utilized the services of a medical officer at Ridge Hospital, such that any patient at risk for impairment was flagged and referred for follow-up.

\section{Data analysis}

Data were analysed using Stata (version 14.1). Summary statistics were performed to create demographic, psycho-behavioural and neurocognitive profiles of participants. Correlation and linear regression analyses were done to determine associations among demographic, psycho-behavioural factors and neurocognitive performance. Receiver Operating Characteristic (ROC) analysis was used to assess how well assessment tools could identify risks for NCI. The IHDS has been designed to flag for HIV-associated dementia, but because the specific cut-offs for different types of HAND are not specified by the IHDS, this study classified NCI risk as a score below the IHDS threshold of $11^{22}$.

\section{Results}

\section{Demographic Profile}

Data from 104 participants were collected during the study period. The demographic characteristics are presented in Table 1 . The mean age of the group was 37.15 \pm 10.06 years. Eighty-two $(78.85 \%)$ of the participants were females, with $67(64.4 \%)$ attaining a minimum of secondary school education and $87(83.7 \%)$ currently employed. The average number of years living with HIV (as of 2017) was $4.75 \pm 3.84$ years.

The majority of the participants took a combination of ART, with the most commonly prescribed being Lamivudine (78.9\%), Efavirenz (58.6\%), Tenofovir (56.7\%) and Zidovudine (18\%). About $50 \%$ of the participants were prescribed hematinics. 
Table 1: Summary of Participant Characteristics

\begin{tabular}{lc}
\hline Variable & Number (\%) \\
\hline Background Characteristics & \\
\hline Sex & $22(21.2)$ \\
Male & $82(78.8)$ \\
Female & $\mathbf{1 0 4}$ \\
Total & \\
\hline Age & $37.15(10.06)$ \\
Mean (SD) & $22(21.2)$ \\
\hline Educational Level & $67(64.4)$ \\
Basic & $15(14.4)$ \\
Secondary & $\mathbf{1 0 4}$ \\
Tertiary & $87(83.7)$ \\
Total & $17(16.3)$ \\
\hline Employment Status & $\mathbf{1 0 4}$ \\
Employed & \\
Unemployed & \\
Total & $6.75(3.84)$ \\
\hline Years of living with HIV & $6(0)$ \\
Mean (SD) & Mean (SD) \\
\hline Instrumental Activities of Daily Living & $4.00(4.47)$ \\
\hline Psycho-behavioural characteristics & $1.14(3.21)$ \\
CES-D score & $0.086(0.318)$ \\
AUDIT score & \\
ART pills missed in past week & $67.77(40.12$ \\
\hline Neurocognitive performance & $21.08(9.75)$ \\
TMT scores (seconds) & $10.11(1.58)$ \\
Stroop Colour-word score (\# of words) & \\
IHDS total score & \\
\hline & \\
\hline & \\
\hline
\end{tabular}

**. Correlation is significant at the 0.01 level (2-tailed).

\section{Psycho-behavioural Profile}

Summary statistics for the Psycho-behavioural profile can be found in Table 1 . The mean score for symptoms of depression was $4.00 \pm 4.46$. The mean score on the AUDIT scale was $1.14 \pm 3.21$, with $86(83.5 \%)$ of the participants reporting not using any alcohol in the past year. ART adherence was noted to be adequate, with 86 (92.47\%) participants reporting missing none, 6 (6.45\%) participants reporting missing one pill in the past week, and $1(1.08 \%)$ participant reporting missing two pills in the past week. Forgetfulness was the main reason cited for the missed pills. There was no significant association between medication adherence and neurocognitive function $(p>0.05)$. All participants had a mean score of 6 on the instrumental activities of daily living, indicating optimal independent physical functioning.

\section{Neurocognitive Profile}

The mean score on the TMT task was $69.77 \pm 40.12$ seconds. The mean for SCW test was $21.07 \pm 9.75$ words. All 104 participants completed the IHDS scale and received a total score derived from the sum of the three subset tests: motor speed, psychomotor speed, and memory recall. The range of scores was from 0 to 12 , with lower scores indicating poorer performance and a score below 11 flagged for risk of neurocognitive impairment. The mean score in the group was 10.11 \pm 1.58 . The mean IHDS scores of participants on or without Efavirenz were 10.10 and 10.11 respectively, and there was no significant difference in their mean scores $(\mathrm{t}$ value $=0.05656, \mathrm{p}=0.9550)$. 
Factors associated with risk of neurocognitive impairment.

Education (Table 2) and age (Table 3) were the only demographic characteristics that exhibited significant associations with risk of NCI.

Table 4 shows a linear regression plot of NCI risk, using IHDS scores against the psycho-behavioural factors investigated (depressive symptoms, alcohol use, ART). Depressive symptoms as measured by the CES-D showed association with NCI risk. When CES-D scores were included in a linear regression model with education, age and risk of NCI (Table 5), significant associations were observed $(\mathrm{F}$ value $=15.086, \mathrm{df}=102, \mathrm{p}<$ 0.0001).

Table 2: Association between different educational levels and IHDS score.

\begin{tabular}{llccr}
\hline \multirow{2}{*}{ Education } & Educational status & Difference & Std. Error & \multicolumn{1}{c}{ Sig. } \\
& & & & \\
\hline Basic & Secondary & -.816 & .366 & .071 \\
& Tertiary & $-1.971^{*}$ & .498 & $<.0001$ \\
Secondary & Basic & .816 & .366 & .071 \\
& Tertiary & $-1.155^{*}$ & .425 & .021 \\
Tertiary & Basic & $1.971^{*}$ & .498 & $<.0001$ \\
& Secondary & $1.155^{*}$ & .425 & .021 \\
\hline
\end{tabular}

*The mean difference is significant at the 0.05 level

Table 3: Association between age and IHDS score

\begin{tabular}{llcc}
\hline \multicolumn{2}{c}{ Variable } & Age & IHDS score \\
& & & \\
\hline Age & $\begin{array}{l}\text { Pearson } \\
\text { Correlation }\end{array}$ & 1 & 0.391 \\
& Sig. & & $<0.0001$ \\
IHDS score & Pearson & & 1 \\
& Correlation & $-0.391^{* *}$ & \\
& Sig. & $<0.0001$ & \\
\hline
\end{tabular}

**Correlation is significant at the 0.01 level (2-tailed). 
Table 4: Linear regression results for psycho-behavioural factors and risk of neurocognitive impairment

\begin{tabular}{cccccc}
\hline NCI risk & B Coefficient & S.E. & $\mathrm{t}$ & P value & $95 \%$ CI \\
\hline Depression & -0.082 & 0.038 & -2.15 & 0.034 & $-0.158--.006$ \\
Alcohol use & 0.045 & 0.054 & 0.84 & 0.402 & $-0.062-.152$ \\
ART pills missed & 0.387 & 0.527 & 0.74 & 0.464 & $-0.659-1.434$ \\
Constant & 10.335 & 0.220 & 47.3 & $<0.0001$ & $9.898-10.771$ \\
\hline
\end{tabular}

Adjusted $R^{2}=0.0304$ (F value $\left.=1.951, d f=91, p=0.127\right)$

Table 5: Association between Depression, age, educational level and NCI risk

\begin{tabular}{ccccc}
\hline Variable & B Coefficient & S.E. & $\mathrm{t}$ & P value \\
\hline Depression & -0.074 & 0.030 & -2.51 & 0.014 \\
Age & -0.055 & 0.013 & -4.12 & $<0.0001$ \\
Educational level & 0.406 & 0.099 & 0.74 & 0.464 \\
Constant & 10.612 & 0.758 & 13.9 & $<0.0001$ \\
\hline Adjusted $R^{2}=0.0314$ (Fvalue $\left.=15.086, d f=102, p<=0.0001\right)$ & &
\end{tabular}

The association between the neurocognitive test scores (TMT and SCW) and the IHDS was significant $(\mathrm{p}<$ $0.0001)$ for both the TMT $(p=0.011)$ and the SCW (p $=0.002$ ).

Using a Receiver Operating Characteristic (ROC) analysis to determine which variables were accurate in classifying a participant as having an NCI risk, the CES-D
Scale, TMT Score, and SCW were selected and each variable was plotted as a curve of sensitivity (true positive) vs. 1 minus specificity (true negative), with the area under the curve representing the variable's accuracy in NCI risk classification. The TMT, CES-D and the SCW were calculated as having $72.14 \%, 56.45 \%$ and $30.9 \%$ accuracy respectively (Figure 1). 


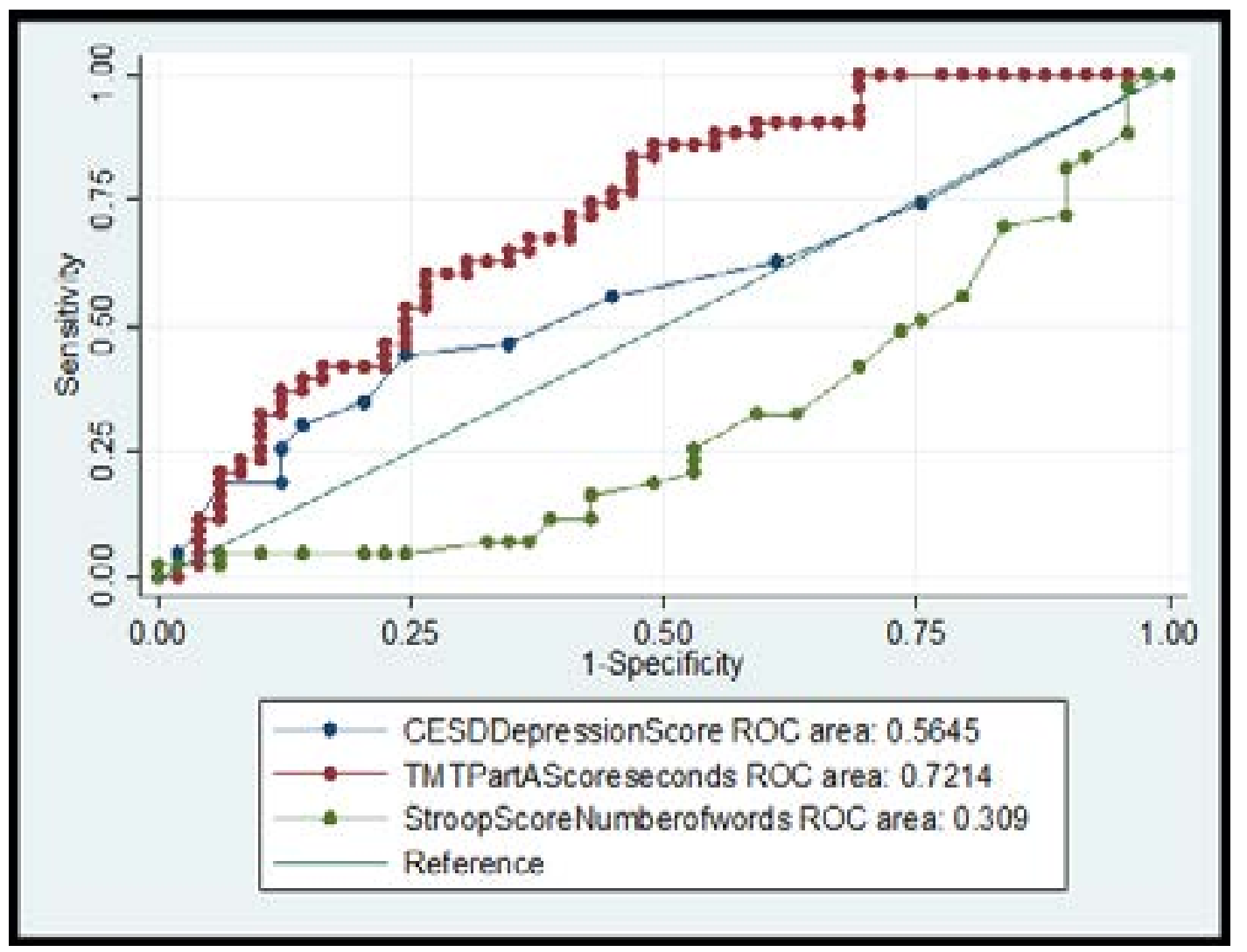

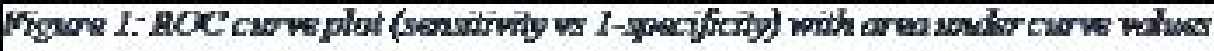

\section{Discussion}

The study assessed neurocognitive performance in a group of people living with HIV. Impairment was assessed on the IHDS, which is an internationally validated scale for assessing HIV-associated dementia and first used in $\mathrm{Uganda}^{20}$. Because the scale originated in another African country, it was believed its application would be well-suited for Ghanaian participants. It has been debated whether IHDS can be effective for diagnosing the milder categories of HAND, with some researchers advocating that the IHDS should be combined with other neurocognitive tests, such as TMT to make it more sensitive to milder $\mathrm{NCI}^{23}$. It was shown that scores on the two tests were correlated with the scores on the IHDS, indicating that the two tests may be good indicators of neurocognitive performance.

About $48 \%$ of the participants had scores slightly below the threshold for risk for NCI using the IHDS. While the IHDS was not enough to diagnose HAND in our sample, the findings are similar to the evidence that almost half of all people living with HIV show some form of risk of cognitive impairment ${ }^{2}$.

Age and education were the demographic factors that related with neurocognitive performance, with longer time to completion on TMT and less words named on SCW being associated with older age. There have been findings from diffuse tensor imaging studies that show the integrity of the brain's white matter declining with age, and because cognitive ability was dependent on processes among brain regions, age played a mediating role in cognitive decline ${ }^{24}$. Similarly, a study on a cohort of HIV-positive individuals on ART showed that the odds of neurocognitive impairment increased by nearly $20 \%$ with each decade of advancing age when follow-up assessments were conducted ${ }^{25}$. Significant associations between education and cognitive impairment have been reported due to cognitive reserve, in which an individual is able to resist age-related brain changes or dementia-related pathology at certain levels of education $^{26}$.

Depression is a significant problem encountered by people living with HIV because of its association with severe immunodeficiency and disease progression ${ }^{27}$. In this study, depression was associated with poor neurocognitive performance, which supports previous findings in which depression had the greatest association with neurocognitive $\operatorname{loss}^{28}$. Depression is frequently co-morbid with HIV infection, so it is unclear which illness may contribute to lower neurocognitive perfor- 
mance ${ }^{29}$. The co-occurrence of HIV and depression has been linked to poor clinical outcomes, with the call for effective mental health policies, and efficient and readily accessible mental health services to address the negative impact of depression on HIV outcomes at the community level as well ${ }^{30}$.

In this study, there was no significant association between alcohol use and NCI, with $83.5 \%$ of the participants reporting no alcohol consumption in the past year. A study about alcohol use in older adults in Ghana showed that heavy consumption of alcohol in the Greater Accra Region was generally lower compared to other regions and the lifetime abstinence rate was also found to be higher in urban areas compared to rural ${ }^{31}$. This may account for the high proportion of non-alcohol consumption in these study participants.

The use of ART in HIV management has been crucial in preventing HIV disease progression from worsening, and adherence to these medications help to mitigate the effects of $\mathrm{NCI}^{32,33}$. In this study, medication adherence was not associated with neurocognitive performance, similar to what has been previously reported ${ }^{33}$. There is also evidence that the neurotoxicity of some HIV medications may contribute to cognitive decline in patients with HIV. For example, a study to assess factors associated with cognitive impairment involving asymptomatic HIV+ patients showed that Efavirenz use predicted worse performance on cognitive tests ${ }^{34}$. Considering that some participants in this study were on Efavirenz $(58.6 \%)$, no significant difference was observed in performance on the IHDS for participants on efavirenz and those who were not on the drug.

When the CES-D, TMT and SCW scores were selected for ROC analysis, TMT had the highest discriminability. This suggests that the TMT may be a good diagnostic tool for NCI when considering resource limited settings including the health facilities at the community level as a testing resource for routine screening by community mental health practitioners. The fact that the TMT showed the highest ability to discriminate NCI is in line with previous evidence that the TMT is able to identify milder forms of HAND as well as detect a wide range of $\mathrm{HAND}^{23}$. For example, a study using TMT in a cognitive battery for individuals with advanced HIV detected HAND with $81.1 \%$ sensitivity, $69.9 \%$ specificity, and $81 \%$ positive predictive value ${ }^{35}$. These observations may be due to the fact that TMT is an assessment of complex attention which is likely what gets compromised in HIV infection of the central nervous system. We acknowledge that a limitation to this study was the absence of a control group for comparison. Another limitation of this study is the lack of assessment for other potential clinical and behavioural modifiers of cognition, such as, viral diseases and use/abuse of additional substances other than alcohol. In addition, using a self-report measure for variables such as alcohol use and medication adherence may be prone to bias since some participants may want to provide socially acceptable responses.

Despite these limitations, this is the first study that has attempted to explore the neurocognitive profile of a sample of Ghanaians living with HIV and on ART. The results from this study should encourage future researchers to do actual prevalence estimates of neurocognitive dysfunction through a comparative study which can provide actual risk estimates. The hope is that this study can bring this subject area into greater prominence for future investigations.

\section{Conclusion}

Some people with HIV in Ghana showed risk of neurocognitive impairment, even when on anti-retroviral medications. This risk was significantly associated with education, age and depressive symptoms. It is therefore important to consider routine neurocognitive screening in HIV-AIDS management to recognize any risks for early interventions. Certain neurocognitive tests like the Trail Making Test could also be considered for measuring neurocognitive impairment in low resource settings.

\section{Conflict of interest disclosure}

None declared.

\section{Acknowledgements}

The authors are grateful to the all the participants and staff at the HIV clinic of the Ridge Hospital in Accra, Ghana.

\section{References}

1. Ousman SS, Kubes P. Immune surveillance in the central nervous system. Nat Neurosci. 2012;15(8):1096. doi: 10.1038/nn.3161

2. Clifford DB, Ances BM. HIV-Associated Neurocognitive Disorder (HAND). Lancet Infect Dis. 2013;13(11):976-86. doi: 10.1016/S14733099(13)70269-X.

3. Habib AG, Yakasai AM, Owolabi LF, Ibrahim A, Habib ZG, Gudaji M, et al. Neurocognitive impairment in HIV-1-infected adults in Sub-Saharan Africa: A systematic review and meta-analysis. Int J Infect Dis. 2013;17(10):e820-31. doi:10.1016/j.ijid.2013.06.011

4. Hardy DJ, Vance DE. The neuropsychology of HIV/ 
AIDS in older adults. Neuropsychol Rev. 2009;19(2):26372. doi: 10.1007/s11065-009-9087-0

5. Vally Z. HIV-associated neurocognitive disorders. South African J Psychiatry. 2011;17(4):98-102. doi. org/10.4102/sajpsychiatry.v17i4.294

6. Robertson K, Liner J, Hakim J, Sankalé J-L, Grant I, Letendre S, et al. NeuroAIDS in Africa. J Neurovirol. 2010;16(3):189-202. doi: 10.3109/13550284.2010.489597

7. Rosca EC, Rosca O, Simu M, Chirileanu RD. HIV-associated Neurocognitive Disorders: A Historical Review. Neurologist. 2012;18(2). doi: 10.1097/ NRL.0b013e318247bc7a

8. Kanmogne GD, Kuate CT, Cysique L a, Fonsah JY, Eta S, Doh R, et al. HIV-associated neurocognitive disorders in sub-Saharan Africa: a pilot study in Cameroon. BMC Neurol. 2010;10:60. doi: 10.1186/14712377-10-60

9. Chibanda D, Benjamin L, Weiss HA, Abas M. Mental, Neurological, and Substance Use Disorders in People Living With HIV/AIDS in Low- and Middle-Income Countries. JAIDS J Acquir Immune Defic Syndr. 2014;67:S54-67. doi: 10.1097/QAI.0000000000000258 10. Ghana AIDS Commission. Country AIDS RESPONSE PROGRESS REPORT-GHANA. Ghana AIDS Commission. 2015.

11. Heaton RK, Clifford DB, Franklin DR, Woods SP, Ake C, Vaida F, et al. HIV-associated neurocognitive disorders persist in the era of potent antiretroviral therapy: CHARTER Study. Neurology. 2010;75:2087-96 PubMed . doi: 10.1212/WNL.0b013e318200d727

12. Ibrahim A, Habib AG, Yakasai AM, Owolabi LF, Habib ZG, Gudaji M, et al. Neurocognitive impairment (NCI) in HIV-1 infected adults in Sub-Saharan Africa: A systematic review and meta-analysis. J Neurol Sci. 2013;333:e294. doi: 10.1016/j.jns.2013.07.1107

13. Bonevski B, Randell M, Paul C, Chapman K, Twyman L, Bryant J, et al. Reaching the hard-to-reach: a systematic review of strategies for improving health and medical research with socially disadvantaged groups. BMC Med Res Methodol. 2014;14(1):42. doi: 10.1186/1471-2288-14-42

14. Radloff LS. The CES-D scale: A self-report depression scale for research in the general population. Appl Psychol Meas. 1977;1(3):385-401 PubMed. doi:10.1177/014662167700100306

15. Reinert DF, Allen JP. The alcohol use disorders identification test (AUDIT): a review of recent research. Alcohol Clin Exp Res. 2002;26(2):272-9. org/10.1111/j.1530-0277.2002.tb02534.x

16. Godin G, Gagné C, Naccache H. Validation of a self-reported questionnaire assessing adherence to antiretroviral medication. AIDS Patient Care STDS. 2003;17(7):325-32. 10.1089/108729103322231268

17. Cangoz B, Karakoc E, Selekler K. Trail Making Test: Normative data for Turkish elderly population by age, sex and education. J Neurol Sci. 2009;283(1-2 PubMed ):73-8. doi: 10.1016/j.jns.2009.02.313. doi: 10.1016/j. acn.2003.09.003

18. Homack S, Riccio CA. A meta-analysis of the sensitivity and specificity of the Stroop Color and Word Test with children. Arch Clin Neuropsychol. 2004;19(6):725-43. doi: 10.1016/j.acn.2003.09.003

19. Franzen MD, Tishelman AC, Sharp BH, Friedman AG. An investigation of the test-retest reliability of the stroop colorword test across two intervals. Arch Clin Neuropsychol. 1987;2(3):265-72. doi.org/10.1016/08876177(87)90014-X

20. Dang C, Wei B, Long J, Zhou M, Han X, Zhao T. Validity of the International HIV Dementia Scale as Assessed in a Socioeconomically Underdeveloped Region of Southern China: Assessing the Influence of Educational Attainment. Int J Infect Dis. 2015;33:56-61. doi: 10.1016/j.ijid.2014.12.042

21. Shelkey M, Wallace M. Katz Index of Independence in Activities of Daily Living (ADL). Hartford Int J Older People. 2012;2(3):204-212.

22. Sacktor NC, Wong M, Nakasujja N, Richard L, Selnes OA, Musisi S, et al. The International HIV Dementia Scale : a new rapid screening test for HIV dementia. AIDS. 2005;19(13):1367-74. PMID:16103767

23. Chalermchai T, Valcour V, Sithinamsuwan P, Pinyakorn S, Clifford D, Paul RH, et al. Trail Making Test A improves performance characteristics of the International HIV Dementia Scale to identify symptomatic HAND. J Neurovirol. 2013;19(2):137-43. doi: 10.1007/ s13365-013-0151-4

24. Bennett, Ilana J. DJM. Disconnected Aging: Cerebral White Matter Integrity and Age- Related Differences in Cognition. Neuroscience. 2014;276:187-205 PubMed . doi: 10.1016/j.neuroscience.2013.11.026

25. Coban H, Robertson K, Smurzynski M, Krishnan $\mathrm{S}$, Wu K, Bosch RJ, et al. Impact of aging on neurocognitive performance in previously antiretroviral-naïve HIV+ individuals on their first suppressive regimen. AIDS. 2017. doi: 10.1097/QAD.0000000000001523.

26. Stern Y. Cognitive reserve in ageing and Alzheimer's disease. Lancet Neurol. 2012 Nov;11(11):1006-12. doi: 10.1016/S1474-4422(12)70191-6.

27. Pinheiro CAT, Souza LDM, Motta JVS, Kelbert EF, Souza MS, Martins CSR, et al. Depression and diagnosis of neurocognitive impairment in HIV-positive patients. 
Brazilian J Med Biol Res. 2016;49(10). doi: 10.1590/1414431X20165344

28. Troncoso FT, Conterno LDO. Prevalence of neurocognitive disorders and depression in a Brazilian HIV population. Rev Soc Bras Med Trop. 2015;48(4):390-8. doi: 10.1590/0037-8682-0034-2015

29. Thames AD, Becker BW, Marcotte TD, Hines LJ, Foley JM, Ramezani A, et al. Depression, Cognition, and Self-Appraisal of Functional Abilties in HIV: An Examination of Subjective Appraisal Versus Objective Performance. Clin Neuropsychol. 2011;25(2):224-43. doi: 10.1080/13854046.2010.539577.

30. Choi SKY, Boyle E, Cairney J, Gardner S, Collins EJ, Bacon J, et al. Adequacy of mental health services for HIV-positive patients with depression: Ontario HIV Treatment Network Cohort Study. PLoS One. 2016;11(6):e0156652. doi: 10.1371/journal. pone.0156652. eCollection 2016.

31. Yawson AE, Welbeck J, Agyenim BJ, Mensah G, Minicuci $\mathrm{N}$ et al. Sociodemographic and Socioeconomic Correlates of Alcohol Use among Older Adults in Ghana. J Alcohol Drug Depend. 2015;03(03):202. doi:10.4172/2329-6488.1000202
32. Saylor D, Dickens AM, Sacktor N, Haughey N, Slusher B, Pletnikov M, et al. HIV-associated neurocognitive disorder - pathogenesis and prospects for treatment. Natl Rev Neurol. 2016;12(4):234-48. doi: 10.1038/ nrneurol.2016.27

33. Andrade ASA, Deutsch R, Celano S, Duarte NA, Marcotte TD, Umlauf A, et al. Relationships Among Neurocognitive Status, Medication Adherence Measured by Pharmacy Refill Records, and Virologic Suppression in HIV-infected Persons. J Acquir Immune Defic Syndr. 2013 Mar 1;62(3):282-92. doi: 10.1097/QAI. 0b013e31827ed678

34. Ciccarelli N, Fabbiani M, Di Giambenedetto S, Fanti I, Baldonero E, Bracciale L, et al. Efavirenz associated with cognitive disorders in otherwise asymptomatic HIV-infected patients. Neurology. 2011;76(16):1403-9. doi: 10.1212/WNL.0b013e31821670fb

35. Cysique LA, Murray JM, Dunbar M, Jeyakumar V, Brew BJ. A screening Algorithm for HIV - Associated Neurocognitive Disorders. 2010;642-9. doi: 10.1111/j.14681293.2010.00834.x 